\title{
Metabolic adaptation following massive weight loss is related to the degree of energy imbalance and changes in circulating leptin
}

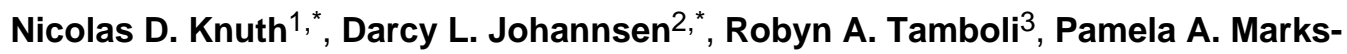 \\ Shulman ${ }^{3}$, Robert Huizenga ${ }^{4}$, Kong Y. Chen ${ }^{1}$, Naji N. Abumrad ${ }^{3}$, Eric Ravussin ${ }^{2}$, and Kevin \\ D. Hall ${ }^{1, \dagger}$ \\ ${ }^{1}$ National Institute of Diabetes and Digestive and Kidney Diseases, National Institutes of Health, \\ Bethesda, MD 20892 \\ ${ }^{2}$ Pennington Biomedical Research Center, Baton Rouge, LA 70808 \\ ${ }^{3}$ Vanderbilt University School of Medicine, Nashville, TN, 37232 \\ ${ }^{4}$ University of California-Los Angeles, Los Angeles, CA 90024
}

\begin{abstract}
Objective-To measure resting metabolic rate (RMR) and body composition changes in obese subjects following massive weight loss achieved via bariatric surgery or calorie restriction plus vigorous exercise.
\end{abstract}

Design and Methods-We compared changes in body composition and RMR in 13 pairs of obese subjects retrospectively matched for sex, body mass index, weight and age that underwent either Roux-en-Y gastric bypass surgery (RYGB) or participated in "The Biggest Loser" weight loss competition (BLC).

Results-Both groups had similar final weight loss (RYGB: $40.2 \pm 12.7 \mathrm{~kg}, \mathrm{BLC}$ : $48.8 \pm 14.9$ $\mathrm{kg} ; p=0.14$ ); however, RYGB lost a larger proportion of their weight as fat-free mass (FFM) (RYGB: $30 \pm 12 \%$, BLC: $16 \pm 8 \%$ [ $p<0.01]$ ). In both groups, RMR decreased significantly more than expected based on measured body composition changes. The magnitude of this metabolic adaptation was correlated with the degree of energy imbalance $(r=0.64, p=0.003)$ and the decrease in circulating leptin $(r=0.61, p=0.006)$.

Conclusions-Calorie restriction along with vigorous exercise in BLC participants resulted in preservation of FFM and greater metabolic adaption compared to RYGB subjects despite comparable weight loss. Metabolic adaptation was related to the degree of energy imbalance and the changes in circulating leptin.

Users may view, print, copy, and download text and data-mine the content in such documents, for the purposes of academic research, subject always to the full Conditions of use:http://www.nature.com/authors/editorial_policies/license.html\#terms

${ }^{\dagger}$ To whom correspondence should be addressed: Kevin D. Hall, Ph.D., National Institute of Diabetes \& Digestive \& Kidney Diseases, National Institutes of Health, 12A South Drive, Room 4007, Bethesda, MD 20892, kevinh @niddk.nih.gov.

*These authors contributed equally to the research and manuscript.

Conflicts of Interest Statement

No authors have conflicts of interest. 


\section{Keywords}

energy metabolism; weight loss; body composition; leptin; bariatric surgery

\section{Introduction}

The prevalence of Class III obesity (i.e., body mass index $>40 \mathrm{~kg} / \mathrm{m}^{2}$ ) is increasing rapidly (1) along with the risk of cardiovascular disease, diabetes, some forms of cancer, and several other chronic diseases (2). Bariatric surgery is an increasingly popular and effective treatment (3) that results in massive weight loss leading to marked improvements in insulin sensitivity, cardio-metabolic health, and reduced mortality $(4,5,6,7)$. However, bariatric surgery patients often experience a disproportionate loss of fat-free mass (FFM) $(8,9,10)$ which may lead to functional deficits, especially with advancing age $(11,12)$. Disproportionate reduction of FFM may also suppress resting metabolic rate (RMR) since FFM comprises the metabolically active tissues of the body (13) and may thereby predispose to weight regain (14).

Few lifestyle interventions can match the degree of weight loss that is achieved through bariatric surgery. This fact makes it difficult to assess the effects of bariatric surgery on energy metabolism and body composition in comparison to a group with similar weight loss achieved via lifestyle intervention alone. The current study aims to investigate changes in body composition, RMR, and cardio-metabolic health in class III obese subjects following massive weight loss achieved with vigorous exercise and self-selected diet restriction as part of "The Biggest Loser" competition (BLC) (15) compared to retrospectively pair-matched subjects following Roux-en-Y gastric bypass surgery (RYGB) (10).

We hypothesized that RYGB would lead to a greater relative loss of FFM compared to BLC participants due to the vigorous exercise of the BLC group. We further hypothesized that both groups would experience a metabolic adaptation, with RMR decreasing greater than expected based on the observed body composition changes and that the magnitude of metabolic adaptation would be related to the degree of energy imbalance and changes in circulating leptin and thyroid hormones.

\section{Methods and Procedures}

\section{Bariatric Surgery}

As previously described (10), RYGB patients were admitted to the Vanderbilt Clinical Research Center on three separate occasions: preoperatively, 6 months postoperatively, and 12 months postoperatively. Patients were under no dietary restrictions prior to the preoperative study visit but following surgery they were counseled to transition from a liquid diet to a balanced diet of $900-1000 \mathrm{kcal} / \mathrm{d}$ containing $70 \mathrm{~g}$ of protein and were encouraged to exercise. The study was approved by the Vanderbilt University Institutional Review Board (\#040572). 


\section{Biggest Loser Intervention}

As previously described (15), BLC participants were housed on a ranch near Los Angeles, CA where they engaged in 90 minutes per day (6d/wk) of directly supervised vigorous circuit training and/or aerobic training and were encouraged to exercise up to an additional 3 $\mathrm{h} / \mathrm{d}$. Participants prepared their own food and were free to eat as desired, but were advised to avoid fats, sugars, and processed grains and consume a calorie restricted diet consisting of low-fat sources of protein and dairy, fresh fruits and vegetables. Participants were instructed to aim for a calorie ratio of protein:carbohydrate:fat of 30:45:25 and advised to consume greater than $70 \%$ of their baseline RMR (16). The study was approved by the Institutional Review Board of Cedars Sinai Medical Center (\#8967) and the Pennington Biomedical Research Center.

\section{Measurements}

Body composition was determined by dual energy x-ray absorptiometry (iDXA; GE Lunar, Madison, WI) and FFM and fat mass (FM) were calculated from weight and whole-body percent fat using the thick scan mode. All participants' supine body width exceeded the dimensions of the scan window and were analyzed using the iDXA MirrorImage ${ }^{\mathrm{TM}}$ application (17). RMR was measured using indirect calorimetry (BLC: Max II metabolic cart, AEI Technologies, Naperville, FL; RYGB: Medgraphics Ultima metabolic cart, Medical Graphics Corporation, St. Paul, MN and TrueOne metabolic cart, ParvoMedics, Sandy, UT). Following a 12-hour fast, participants rested supine in a quiet, darkened room for 30 minutes before measurements of $\mathrm{VO}_{2}$ and $\mathrm{VCO}_{2}$ for 20 minutes with the last 15 minutes used to determine RMR. Body composition and RMR were also measured in 9 of the RYGB patients at 6 months postoperatively.

\section{Biochemical Assays}

Blood samples from overnight fasted BLC participants were analyzed by a commercial laboratory (West Coast Clinical Laboratories, Van Nuys, CA). The chemistry panel was measured on a Beckman Synchron CX5CE or CX9PRO. Insulin was determined by radioimmunoassay, and leptin and adiponectin concentrations were measured using a commercially available kit (Millipore, St. Charles, MO). Analysis of overnight fasted blood samples from RYGB subjects was performed at Vanderbilt University Medical Center. Glucose was measured at the bedside using the glucose oxidase method (Beckman Glucose Analyzer, Fullerton, CA). Insulin and leptin were determined by radioimmunoassay and adiponectin by multiplex immunoassay (Millipore, St. Charles, MO). Triglycerides and total, HDL, and LDL cholesterol were assayed with ACE reagents and instrumentation (Alfa Wassermann, Caldwell, NJ). Insulin resistance was calculated using the homeostasis model assessment of insulin resistance (HOMA-IR) using fasting measurements of glucose and insulin (18). In both BLC and RYGB samples, thyroid panel (T3, T4, TSH) was measured by immunoassay with chemiluminescent detection (Millipore Corporation, Billerica, MA). Samples from only 9 of the 13 pairs were available for analysis due to a lack of sufficient sample for 4 RGYB participants. 


\section{Statistical Analysis}

Data are expressed as mean \pm SD. Analyses were performed using Intercooled Stata version 10.0 (Stata Corp, LP, College Station, TX). BLC and RYGB subjects were retrospectively matched on the basis of sex and baseline body weight, BMI, and age using the statistical software command "optmatch2" which creates sets of subjects with the smallest possible dissimilarities. Linear regression analysis was used to generate an equation for RMR using baseline data from all BLC $(n=16)$ and RYGB $(n=26)$ subjects. The independent variables for the regression analysis were FFM, FM, age, and group. The group term was included because different metabolic carts were used at each site resulting in an environment consistent within groups but different between groups.

At follow-up, differences between the measured and predicted RMR defined the magnitude of metabolic adaptation which was considered to be present if the RMR residuals were significantly different from zero (15). Associations between the degree of metabolic adaptation, energy imbalance, percent change in leptin, average rate of weight loss, and thyroid hormones were examined and the Bonferroni correction was used to adjust for multiple comparisons. The average degree of energy imbalance was calculated from the measured rates of change of FM and FFM along with their respective energy densities of 9.4 $\mathrm{kcal} / \mathrm{g}$ and $1.2 \mathrm{kcal} / \mathrm{g}(19)$. Statistical analysis of 6-month RYGB data in comparison to baseline and 12 months was limited to data from the same 9 RYGB patients at all time points.

\section{Results}

\section{Study Participants}

Twenty-two female and 4 male RYGB patients had complete data at baseline and 12 months with an age range of $27-58$ years $\left(43 \pm 10 \mathrm{y}\right.$ ), a mean BMI of $46.9 \pm 7.1 \mathrm{~kg} / \mathrm{m}^{2}$ and a mean body weight of $130.6 \pm 23.6 \mathrm{~kg}$, half of which was fat (50 $\pm 5 \%$ ). The BLC participants were 9 women and 7 men ranging in age from 20 to 56 years $(33 \pm 10 \mathrm{y})$ and, similar to the RYGB cohort, they were Class III obese at baseline with a mean BMI of $49.4 \pm 9.4 \mathrm{~kg} / \mathrm{m}^{2}$ and body weight of $149.2 \pm 38.0 \mathrm{~kg}$, of which nearly half was fat $(49 \pm 5 \%)$. Software-aided matching of the two cohorts for sex, body weight, BMI, and age resulted in 13 pairs of subjects ( 9 women, 4 men) with similar baseline body weight (BLC: $140 \pm 36 \mathrm{~kg}, p=0.68$, RYGB: $135 \pm 28 \mathrm{~kg}$ ), BMI (BLC: $47.6 \pm 9.5 \mathrm{~kg} / \mathrm{m}^{2}$, RYGB: $47.0 \pm 7.6 \mathrm{~kg} / \mathrm{m}^{2}, p=0.87$ ), and age (BLC: $32 \pm 11 \mathrm{yr}, \mathrm{RYGB}: 39 \pm 9 \mathrm{yr}, p=0.14$ ) (Table 1). There were no significant differences in body weight, BMI, or age, at baseline between RYGB subjects with or without 6 month data (18 with/8 without).

\section{Body Weight and Composition}

BLC participants lost weight more rapidly than RYGB patients. At 7 months, BLC participants lost an average of $48.8 \pm 14.9 \mathrm{~kg}$ whereas RYGB patients did not achieve a similar amount of weight loss until 12 months after surgery $(40.2 \pm 12.7 \mathrm{~kg}, p=0.14)$. At 6 months RYGB patients lost $35.6 \pm 8.7 \mathrm{~kg}$, of which $29.2 \pm 13.5 \%$ was from loss of FFM. Therefore, most of the FFM loss in RYGB patients occurred in the early post-surgery period. For RYGB patients with data at both 6 and 12 months, weight loss at 6 months was 
significantly less than at 12 months $(35.6 \pm 8.7 \mathrm{~kg}$ vs. $44.3 \pm 11.7 \mathrm{~kg}, p<0.005)$. Despite similar weight loss at 7 months for BLC compared with 12 months for RYGB, the BLC subjects demonstrated a relative preservation of FFM with only $16.4 \pm 8.1 \%$ of their weight loss from FFM. In contrast, the FFM proportion of weight loss in the RYGB patients was $29.6 \pm 11.9 \%$ and was significantly greater than BLC participants $(p<0.01)$ (Table 1 and Figure 1).

\section{Resting Metabolic Rate}

At the end of the study, metabolic rate was suppressed to a greater degree in BLC participants compared to RYGB patients despite similar amounts of weight loss. In BLC, measured RMR was reduced by over $600 \mathrm{kcal} / \mathrm{d}(p<0.001)$, whereas it decreased by $\sim 300$ $\mathrm{kcal} / \mathrm{d}(p<0.001)$ in RYGB patients (Table 1). In RYGB patients with 6 and 12 month data, average RMR decreased significantly from baseline to 6 months $(2194 \pm 307 \mathrm{kcal} / \mathrm{d}$ to 1754 $\pm 377 \mathrm{kcal} / \mathrm{d}, p<0.005$ ), and was maintained at 12 months despite continued weight loss $(1872 \pm 211 \mathrm{kcal} / \mathrm{d}, p=0.12)$.

To investigate how much of the observed suppression in metabolic rate could be accounted for by body composition change, we used the baseline RMR data to generate a prediction equation as a function of FFM, FM, age, and group. The residual between the measured and predicted RMR defined the degree of metabolic adaptation. The predicted average RMR in BLC participants at 7 months was $2275 \pm 330 \mathrm{kcal} / \mathrm{d}$ which was $419 \pm 169 \mathrm{kcal} / \mathrm{d}$ more than measured $(p<0.001)$, indicating that significant metabolic adaptation was present at the end of the weight loss period. In RYGB patients, there was a greater than expected decrease in RMR of $201 \pm 182 \mathrm{kcal} / \mathrm{d}(p<0.05)$ at 6 months; however, at 12 months there was no significant difference between measured and predicted RMR $(8 \pm 191 \mathrm{kcal} / \mathrm{d}, p=0.89)$. Therefore, metabolic adaptation had dissipated in the RYGB patients at 12 months despite a similar amount of weight loss compared with BLC participants at 7 months (Figure 2).

The average energy imbalance was significantly greater in BLC compared to RYGB (Table 1) and was positively associated with the degree of metabolic adaptation in combined BLC and RYGB at the end of the study $(r=0.64, p=0.003)$. The degree of metabolic adaptation also correlated with the average rate of weight loss $(r=0.61, p=0.006)$. Furthermore, the percent change in circulating leptin at the end of the study was found to be positively associated with metabolic adaptation $(r=0.61, p=0.006)$. While the observed changes in leptin may have been greater than expected based on the observed body composition changes, we found no evidence that the residual leptin changes were correlated with metabolic adaptation (data not shown). The correlations between metabolic adaptation and energy imbalance ( $r=0.55, p=0.004$ (Figure 3A), rate of weight loss $(r=0.53, p=0.007$ ), and change in leptin $(r=0.47, p=0.03)$ (Figure 3A) persisted when including RYGB data at 6 months.

\section{Cardio-metabolic Profile}

Both interventions resulted in similar improvements in insulin sensitivity and cardiometabolic profile despite some differences at baseline (Table 2). Fasting glucose concentration was similar between BLC and RYGB at baseline, but fasting insulin 
concentration was lower in the BLC group. Together, this resulted in a significantly lower calculated HOMA-IR in BLC compared to RYGB, indicating that BLC participants were more insulin sensitive at baseline. Fasting glucose and insulin decreased with weight loss to within the normal range in both BLC and RYGB, although the final mean concentrations were lower in BLC than in RYGB (Table 2). As a consequence, both BLC and RYGB demonstrated improved insulin sensitivity as HOMA-IR decreased by over $50 \%$ in both groups. Blood pressure was similar between BLC and RYGB at baseline and significantly decreased with weight loss in BLC, with a trend towards improved systolic pressure in RYGB $(p=0.08)$.

Triglycerides, adiponectin, and C-peptide concentrations were lower in BLC than RYGB at baseline; however, cholesterol, HDL, LDL, and leptin were similar (Table 2). While circulating triglycerides decreased significantly with weight loss in both groups, total cholesterol tended to increase in BLC and consisted of increases in both HDL and LDL components. In RYGB, total cholesterol decreased mainly as a result of lower LDL. As expected with weight loss, leptin decreased significantly in both BLC and RYGB, but the decrease was greater in BLC with the final leptin concentration being $80 \%$ lower in BLC compared to RYGB despite similar FM. Thyroid profile was measured in 9 pairs of subjects and was similar at baseline between BLC and RYGB. Triiodothyronine significantly decreased in BLC after weight loss but not in RYGB. The change in $\mathrm{T}_{3}$ concentration after weight loss in the combined group was positively associated with the average negative energy balance $(\mathrm{r}=0.84, p<0.001)$ and rate of weight loss $(\mathrm{r}=0.78, p<0.01)$, but in contrast to our hypothesis there was no significant association between changes in $\mathrm{T}_{3}$ and metabolic adaptation $(\mathrm{r}=0.58, p=0.41)$. No significant changes occurred in thyroxin and thyroid stimulating hormone were observed in BLC or RYGB after weight loss.

\section{Discussion}

We observed similarly massive weight loss in both BLC participants and RYGB patients over 7 and 12 months, respectively. On average, both groups lost in excess of 30\% of their initial weight with a corresponding resolution of their Class III obesity and significant improvements in cardiometabolic profile. Insulin sensitivity doubled, circulating triglycerides were cut in half, and blood pressure tended to decrease in both groups.

An important objective during weight loss is to reduce body fat while minimizing loss of FFM to maintain optimal metabolic and physical functioning. Limited data suggest that adding exercise to a weight loss program may help spare FFM, but most previous studies have investigated only modest weight loss $(20,21,22)$. Here, we demonstrated that in the context of rapid and massive weight loss, BLC participants preserved their FFM with only $16 \%$ of total weight loss from FFM compared with $30 \%$ in matched RYGB patients who lost a similar amount of weight. The BLC participants were advised to consume a diet comprising $30 \%$ of energy from protein, and, given their energy intake of $\sim 1600 \mathrm{kcal} / \mathrm{d}$ over the course of the intervention (23), they may have consumed more protein than was recommended to the RYGB group (about $120 \mathrm{~g} / \mathrm{d}$ vs. $70 \mathrm{~g} / \mathrm{d}$, respectively). Therefore, increased dietary protein may have contributed to the relative maintenance of FFM in BLC vs. RYGB. Furthermore, the BLC participants were more insulin sensitive at baseline which 
may have played a role in preserving FFM. However, the vigorous exercise program likely played a predominant role in preserving FFM in BLC participants due to skeletal muscle hypertrophy (24). Thus, substantial losses of FFM are not a necessary consequence of rapid massive weight loss. Whether incorporating a resistance exercise program or an increase in dietary protein following gastric bypass would result in similar preservation of FFM is a subject worthy of future investigation.

Since FFM comprises the metabolically active lean tissues of the body (13), its relative preservation in BLC participants would be expected to result in better maintenance of RMR compared with RYGB patients (22). However, despite the substantial differences in FFM loss between the groups, RMR decreased to a greater degree in BLC versus RYGB. Furthermore, both groups exhibited a reduction in RMR out of proportion to the decrease in FFM and FM indicating significant metabolic adaptation to weight loss, although metabolic adaptation in RYGB patients only occurred at month 6 during active weight loss.

The mechanisms underlying metabolic adaptation are not clear, but it has been speculated to involve decreases in circulating leptin and thyroid hormones in association with blunted activity of the sympathetic nervous system $(25,26,27,28,29,30)$. In support of this concept, we found that the degree of metabolic adaptation was correlated with the change in leptin as well as the degree of average energy deficit in our subjects. Note that the observed FM changes were included within the RMR prediction model. Therefore, the relationship between changes in leptin and metabolic adaptation was beyond the expected effect of FM loss on RMR. Emerging data suggests that replacement of circulating leptin to pre-weight loss levels reverses metabolic adaptation, perhaps through alterations in mitochondrial content and/or coupling $(31,32)$, and maintenance of thyroid hormone concentrations $(28$, $33)$.

Another possible explanation for the increased metabolic adaptation in BLC participants may be due to the nature of the weight loss competition with significantly decreased energy intake and increased exercise in the days leading up to the final measurements. Such an acute negative energy balance may have led to transient additional suppression of circulating leptin, sympathetic drive, and thyroid function $(27,29,34)$. Some evidence of this possibility includes the larger decrease in leptin in comparison to RYGB and the rise in circulating cholesterol which has been observed following acute starvation $(35,36)$. In contrast, it is likely that RYGB subjects may have been in a state of approximate energy balance at 12 months when metabolic adaptation had dissipated.

Limitations of this study include the differing rates of weight loss in the BLC and RYGB groups and the lack of a weight loss maintenance phase to investigate the subjects in a state of energy balance. Furthermore, the physical activity status of RYGB subjects was not measured, but we believe that their exercise levels were likely substantially lower than BLC subjects. While persistent weight loss is a hallmark of bariatric surgery, significant weight regain is sometimes observed following gastric bypass (37) which has been related to reduced RMR (14). Metabolic adaptation may therefore predispose individuals to weight regain, and BLC participants exhibited a greater metabolic adaptation at 7 months compared to RYGB subjects at 12 months despite similar weight loss. However, the magnitude of the 
energy deficit is likely a main driver of metabolic adaptation, as predicted by mathematical models of human metabolism $(23,38)$. Therefore, the metabolic adaptation in BLC participants would be expected to be greatly attenuated during a weight loss maintenance phase when energy balance is achieved. Indeed, we found that RYGB subjects had no metabolic adaptation at 12 months when their energy imbalance was greatly reduced in comparison to 6 months after surgery.

Despite our attempts to retrospectively match subjects, there were significant differences in several baseline circulating hormones, biochemical and metabolic measurements, possibly due to different assays and equipment used, which may have confounded our interpretation of the differences between the groups. While it would be highly desirable to prospectively match subjects undergoing RYGB and BLC interventions, the opportunistic nature of studying the latter intervention makes this difficult.

An advantage of the intensive lifestyle intervention is that BLC participants had greatly improved physical fitness and were fully capable of maintaining the levels of physical activity characteristic of successful weight loss maintenance $(23,39)$. In contrast, success in achieving a sustained reduction in energy intake and thereby maintaining weight loss in RYGB is likely the result of alterations in appetite, satiety, and food reward systems (40).

In conclusion, we found that an intensive lifestyle intervention consisting of daily vigorous exercise plus self-selected dietary restriction resulted in massive weight loss of similar magnitude to a matched group of subjects following gastric bypass surgery. Both groups showed significant improvements in cardio-metabolic profile and a resolution of Class III obesity. Unlike RYGB patients who lost a substantial amount of FFM, BLC participants partially preserved FFM despite rapid weight loss. However, FFM preservation did not attenuate the reduction of metabolic rate and both groups demonstrated a significant metabolic adaptation to weight loss that was correlated with the energy deficit and changes in leptin.

\section{Acknowledgements}

This research was supported in part by the Intramural Research Program of the NIH, National Institute of Diabetes \& Digestive \& Kidney Diseases (NDK and KDH), the Pennington NORC Center Grant \# P30 DK072476 (to ER), NIDDK grants R01-DK070860 to NNA, DK20593 to the Vanderbilt Diabetes Research and Training Center, DK058404 to the Vanderbilt Digestive Disease Research Center; K01DK89005 to DLJ, and the Vanderbilt CTSA grant 1 UL1 RR024975 from the NCRR. NDK, DLJ, ER, NNA, KYC, and KDH designed the research; NDK, DLJ, RAT, PAMS, NNA, and RH conducted the research; NDK and KDH analyzed data or performed statistical analysis; NDK, DLJ, ER, RH, RAT, and KDH wrote the paper; KDH had primary responsibility for final content.

\section{References}

1. Sturm R, Hattori A. Morbid obesity rates continue to rise rapidly in the United States. Int J Obes (Lond). 2013; 37:889-891. [PubMed: 22986681]

2. Pi-Sunyer FX. The medical risks of obesity. Postgrad Med. 2009; 121:21-33. [PubMed: 19940414]

3. Buchwald H, Oien DM. Metabolic/bariatric surgery Worldwide 2008. Obes Surg. 2009; 19:16051611. [PubMed: 19885707]

4. Adams TD, Gress RE, Smith SC, Halverson RC, Simper SC, Rosamond WD, et al. Long-term mortality after gastric bypass surgery. N Engl J Med. 2007; 357:753-761. [PubMed: 17715409] 
5. Ashrafian H, le Roux CW, Darzi A, Athanasiou T. Effects of bariatric surgery on cardiovascular function. Circulation. 2008; 118:2091-2102. [PubMed: 19001033]

6. Pontiroli AE, Morabito A. Long-term Prevention of Mortality in Morbid Obesity Through Bariatric Surgery. A Systematic Review and Meta-analysis of Trials Performed With Gastric Banding and Gastric Bypass. Ann Surg. 2011

7. Sjostrom L, Narbro K, Sjostrom CD, Karason K, Larsson B, Wedel H, et al. Effects of bariatric surgery on mortality in Swedish obese subjects. N Engl J Med. 2007; 357:741-752. [PubMed: 17715408]

8. Chaston TB, Dixon JB, O'Brien PE. Changes in fat-free mass during significant weight loss: a systematic review. Int J Obes (Lond). 2007; 31:743-750. [PubMed: 17075583]

9. Hall KD. Body fat and fat-free mass inter-relationships: Forbes's theory revisited. Br J Nutr. 2007; 97:1059-1063. [PubMed: 17367567]

10. Tamboli RA, Hossain HA, Marks PA, Eckhauser AW, Rathmacher JA, Phillips SE, et al. Body composition and energy metabolism following Roux-en-Y gastric bypass surgery. Obesity (Silver Spring). 18:1718-1724. [PubMed: 20414197]

11. Miller SL, Wolfe RR. The danger of weight loss in the elderly. The journal of nutrition, health \& aging. 2008; 12:487-491.

12. Villareal DT, Banks M, Siener C, Sinacore DR, Klein S. Physical frailty and body composition in obese elderly men and women. Obes Res. 2004; 12:913-920. [PubMed: 15229329]

13. Muller MJ, Bosy-Westphal A, Kutzner D, Heller M. Metabolically active components of fat-free mass and resting energy expenditure in humans: recent lessons from imaging technologies. Obes Rev. 2002; 3:113-122. [PubMed: 12120418]

14. Faria SL, Kelly E, Faria OP. Energy expenditure and weight regain in patients submitted to Rouxen-Y gastric bypass. Obes Surg. 2009; 19:856-859. [PubMed: 19399563]

15. Johanssen DL, Knuth ND, Huizenga R, Rood J, Ravussin E, Hall KD. Metabolic slowing with massive weight loss despite preservation of fat-free mass. J Clin Endocrinol Metab. 2012; 97:2489-2496. [PubMed: 22535969]

16. Cunningham JJ. Body composition and resting metabolic rate: the myth of feminine metabolism. Am J Clin Nutr. 1982; 36:721-726. [PubMed: 7124675]

17. Rothney MP, Brychta RJ, Schaefer EV, Chen KY, Skarulis MC. Body composition measured by dual-energy X-ray absorptiometry half-body scans in obese adults. Obesity (Silver Spring). 2009; 17:1281-1286. [PubMed: 19584885]

18. Matthews DR, Hosker JP, Rudenski AS, Naylor BA, Treacher DF, Turner RC. Homeostasis model assessment: insulin resistance and beta-cell function from fasting plasma glucose and insulin concentrations in man. Diabetologia. 1985; 28:412-419. [PubMed: 3899825]

19. Hall KD. What is the required energy deficit per unit weight loss? Int J Obes (Lond). 2008; 32:573-576. [PubMed: 17848938]

20. Garrow JS. Exercise in the treatment of obesity: a marginal contribution. Int J Obes Relat Metab Disord. 1995; 19(Suppl 4):S126-S129. [PubMed: 8581088]

21. Redman LM, Heilbronn LK, Martin CK, Alfonso A, Smith SR, Ravussin E. Effect of calorie restriction with or without exercise on body composition and fat distribution. J Clin Endocrinol Metab. 2007; 92:865-872. [PubMed: 17200169]

22. Stiegler P, Cunliffe A. The role of diet and exercise for the maintenance of fat-free mass and resting metabolic rate during weight loss. Sports medicine (Auckland, NZ. 2006; 36:239-262.

23. Hall KD. Diet versus exercise in "the biggest loser" weight loss competition. Obesity (Silver Spring). 2013; 21:957-959. [PubMed: 23404767]

24. Phillips SM. Physiologic and molecular bases of muscle hypertrophy and atrophy: impact of resistance exercise on human skeletal muscle (protein and exercise dose effects). Applied physiology, nutrition, and metabolism = Physiologie appliquee, nutrition et metabolisme. 2009; 34:403-410.

25. Arone LJ, Mackintosh R, Rosenbaum M, Leibel RL, Hirsch J. Autonomic nervous system activity in weight gain and weight loss. Am J Physiol. 1995; 269:R222-R225. [PubMed: 7631897]

26. Leibel RL, Rosenbaum M, Hirsch J. Changes in energy expenditure resulting from altered body weight. N Engl J Med. 1995; 332:621-628. [PubMed: 7632212] 
27. Rosenbaum M, Hirsch J, Murphy E, Leibel RL. Effects of changes in body weight on carbohydrate metabolism, catecholamine excretion, and thyroid function. Am J Clin Nutr. 2000; 71:1421-1432. [PubMed: 10837281]

28. Rosenbaum M, Murphy EM, Heymsfield SB, Matthews DE, Leibel RL. Low dose leptin administration reverses effects of sustained weight-reduction on energy expenditure and circulating concentrations of thyroid hormones. J Clin Endocrinol Metab. 2002; 87:2391-2394. [PubMed: 11994393]

29. Rosenbaum M, Nicolson M, Hirsch J, Murphy E, Chu F, Leibel RL. Effects of weight change on plasma leptin concentrations and energy expenditure. J Clin Endocrinol Metab. 1997; 82:36473654. [PubMed: 9360521]

30. Rosenbaum M, Vandenborne K, Goldsmith R, Simoneau JA, Heymsfield S, Joanisse DR, et al. Effects of experimental weight perturbation on skeletal muscle work efficiency in human subjects. Am J Physiol Regul Integr Comp Physiol. 2003; 285:R183-R192. [PubMed: 12609816]

31. Esterbauer H, Oberkofler H, Dallinger G, Breban D, Hell E, Krempler F, et al. Uncoupling protein-3 gene expression: reduced skeletal muscle mRNA in obese humans during pronounced weight loss. Diabetologia. 1999; 42:302-309. [PubMed: 10096782]

32. Guijarro A, Osei-Hyiaman D, Harvey-White J, Kunos G, Suzuki S, Nadtochiy S, et al. Sustained weight loss after Roux-en-Y gastric bypass is characterized by down regulation of endocannabinoids and mitochondrial function. Ann Surg. 2008; 247:779-790. [PubMed: 18438115]

33. Rosenbaum M, Goldsmith R, Bloomfield D, Magnano A, Weimer L, Heymsfield S, et al. Lowdose leptin reverses skeletal muscle, autonomic, and neuroendocrine adaptations to maintenance of reduced weight. J Clin Invest. 2005; 115:3579-3586. [PubMed: 16322796]

34. Weinsier RL, Nagy TR, Hunter GR, Darnell BE, Hensrud DD, Weiss HL. Do adaptive changes in metabolic rate favor weight regain in weight-reduced individuals? An examination of the set-point theory. Am J Clin Nutr. 2000; 72:1088-1094. [PubMed: 11063433]

35. Ende N. Starvation studies with special reference to cholesterol. Am J Clin Nutr. 1962; 11:270280.

36. Thampy KG. Hypercholesterolaemia of prolonged fasting and cholesterol lowering of re-feeding in lean human subjects. Scandinavian journal of clinical and laboratory investigation. 1995; 55:351357. [PubMed: 7569738]

37. Shah M, Simha V, Garg A. Review: long-term impact of bariatric surgery on body weight, comorbidities, and nutritional status. J Clin Endocrinol Metab. 2006; 91:4223-4231. [PubMed: 16954156]

38. Hall KD. Modeling metabolic adaptations and energy regulation in humans. Annu Rev Nutr. 2012; 32:35-54. [PubMed: 22540251]

39. Wing RR, Phelan S. Long-term weight loss maintenance. Am J Clin Nutr. 2005; 82:222S-225S. [PubMed: 16002825]

40. Shin AC, Berthoud HR. Food reward functions as affected by obesity and bariatric surgery. Int J Obes (Lond). 2011; 35(Suppl 3):S40-S44. [PubMed: 21912387] 


\section{What is already known about this subject}

- Bariatric surgery results in massive weight loss and a substantial loss of fat-free mass (FFM) which may suppress metabolic rate and predispose to weight regain.

- Lifestyle interventions rarely achieve similarly massive weight loss as bariatric surgery, thus making it difficult to compare the effects of these interventions on metabolism and body composition.

- Weight loss typically results in a suppression of resting metabolic rate (RMR) that is often beyond what is expected due to changes in body composition - a phenomenon called metabolic adaptation.

\section{What this study adds}

- We demonstrated that an intensive lifestyle intervention incorporating vigorous exercise preserved FFM compared to bariatric surgery despite losing a similar amount of weight at a faster pace.

- Despite similar weight loss and relative preservation of FFM, RMR decreased to a greater degree at the end of 7 months of the lifestyle intervention compared with 12 months following bariatric surgery.

- Metabolic adaptation was significant in both interventions and was associated with the degree of energy imbalance as well as the change in circulating leptin. 


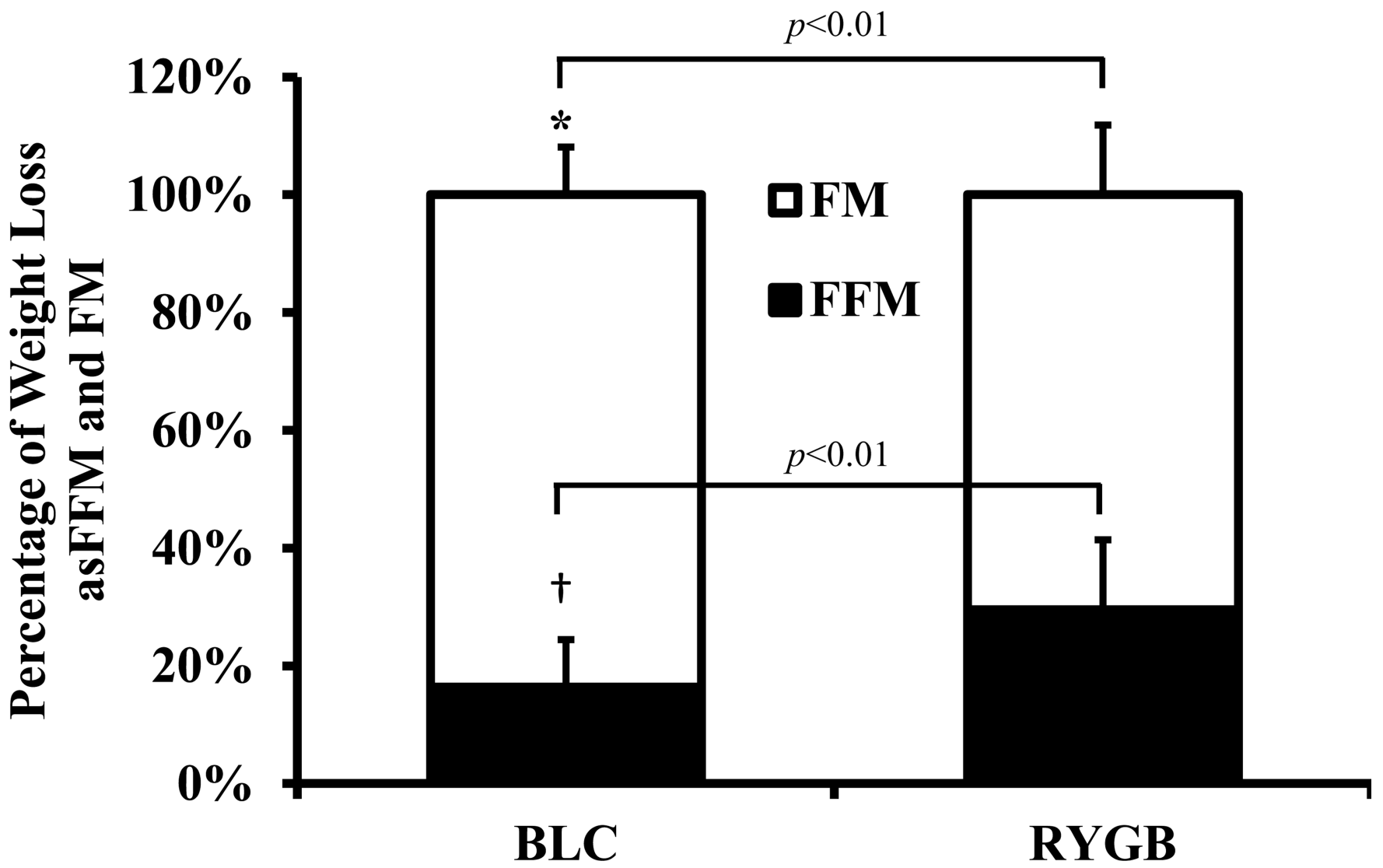

Figure 1.

Percentage of total weight loss from fat mass (FM) and fat-free mass (FFM) in 13 pairmatched BLC and RYGB participants (mean $\pm \mathrm{SD}$ ). $* P<0.01$ BLC percentage of total weight loss from fat mass significantly different from RYGB. $\dagger P<0.01$ BLC percentage of total weight loss from fat-free mass significantly different from RYGB. 


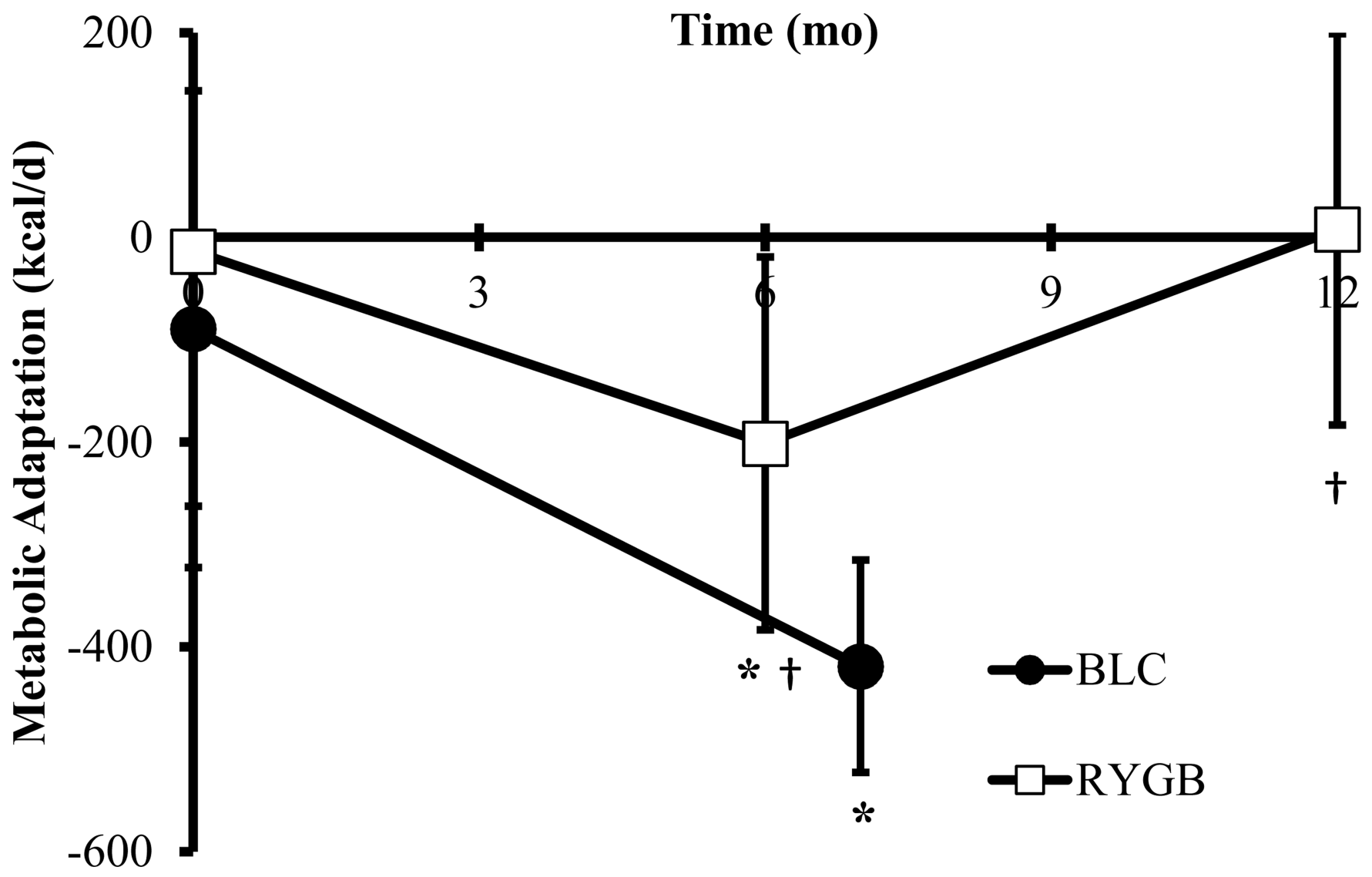

Figure 2.

Metabolic adaptation (measured minus predicted RMR) in BLC $(\bullet)$ at baseline and 7 months; and in RYGB ( $\square$ ) at baseline (n=13), 6 months ( $=9$ ), and 12 months ( $\mathrm{n}=13$ ) (mean $\pm \mathrm{SD}) . * P<0.01$ metabolic adaptation significantly different from zero. $\dagger P<0.05$ compared with BLC 7 month. 
A

\section{Energy Imbalance (kcal/d)}

$\begin{array}{lllllll}-3000 & -2500 & -2000 & -1500 & -1000 & -500 & 0\end{array}$

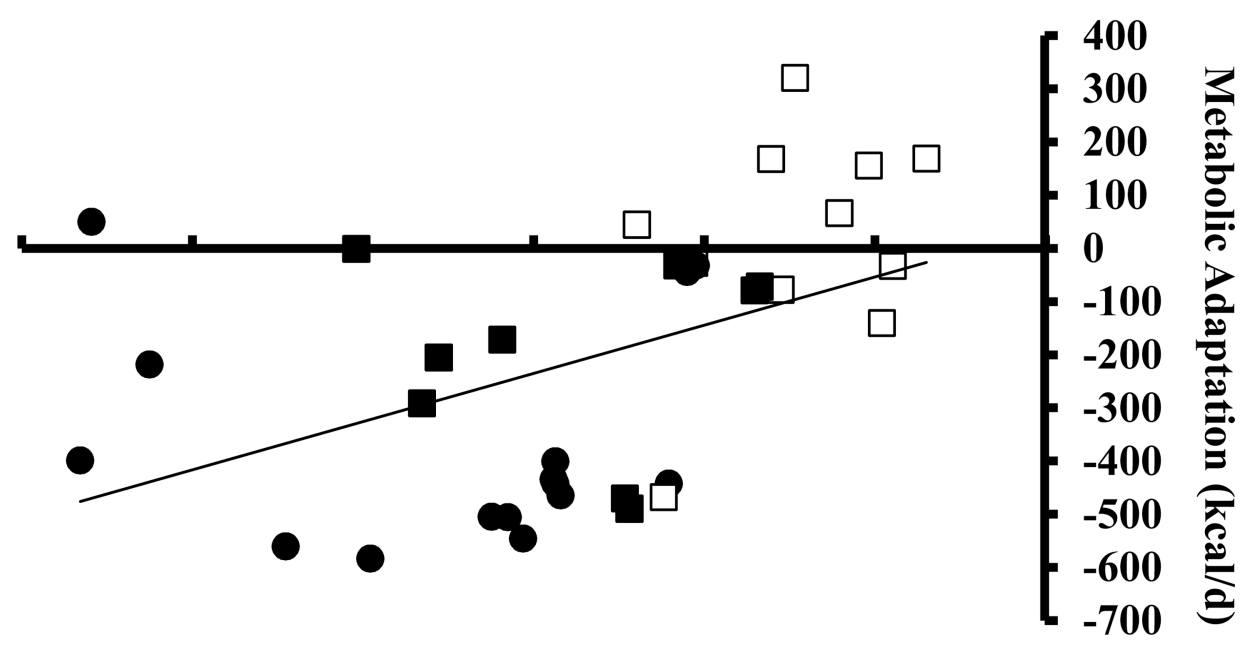

B

\section{Percent Change in Leptin}

$\begin{array}{llllll}-100 \% & -80 \% & -60 \% & -40 \% & -20 \% & 0 \%\end{array}$

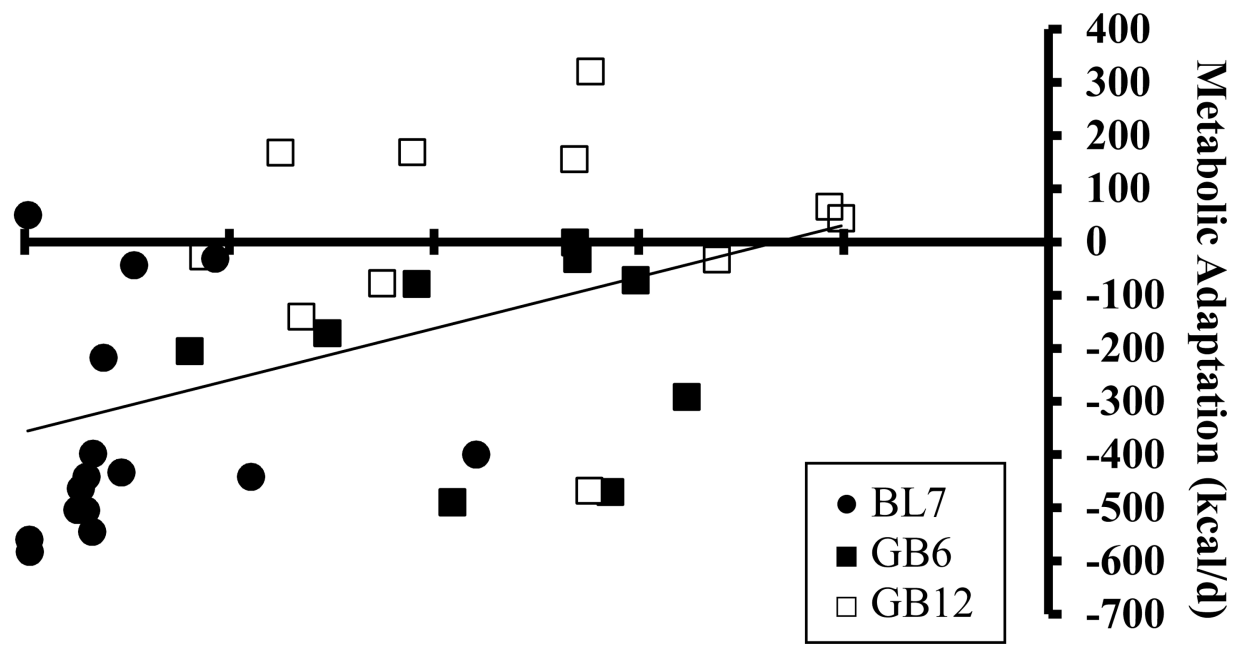

Figure 3.

Correlation between metabolic adaptation and (A) average energy imbalance $(r=0.55, p$ $=0.004)$ and $(\mathrm{B})$ percent decrease in circulating leptin $(r=0.47, p=0.02)$ in BLC at 7

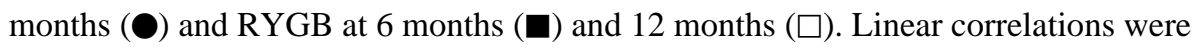
calculated for all data combined. 

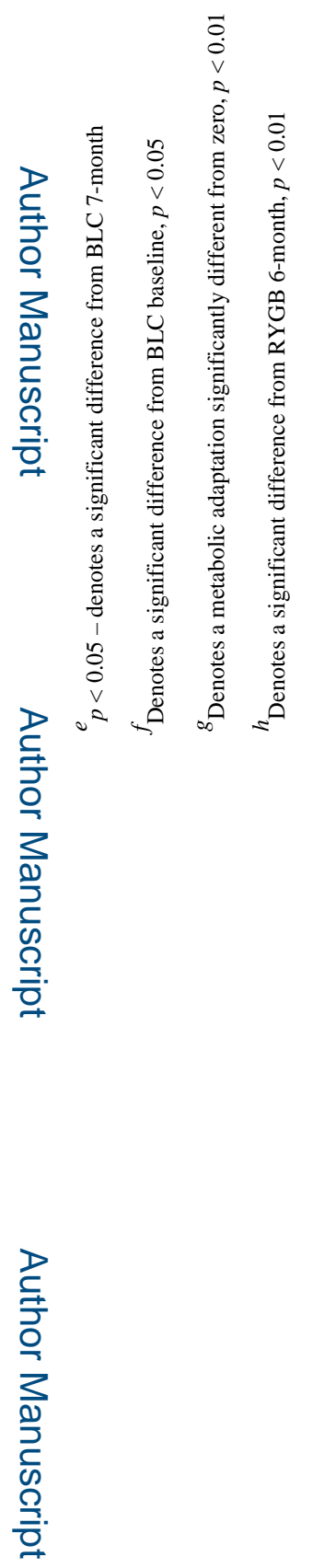

로을

Obesity (Silver Spring). Author manuscript; available in PMC 2015 June 01. 
Table 2

Biochemical and blood pressure measurements in 13 pair-matched BLC and RYGB participants.

\begin{tabular}{|c|c|c|c|c|}
\hline & \multicolumn{2}{|c|}{ BLC $(n=13)$} & \multicolumn{2}{|c|}{ RYGB $(n=13)$} \\
\hline & Baseline & 7 Months & Baseline & 12 Months \\
\hline Glucose (mg/dL) & $97 \pm 17$ & $78 \pm 5^{c}$ & $113 \pm 42$ & $88 \pm 9 d, e$ \\
\hline Insulin $(\mu \mathrm{U} / \mathbf{m L})$ & $8.0 \pm 7.5$ & $4.2 \pm 1.9$ & $25.5 \pm 11.9^{a}$ & $9.2 \pm 3.9^{c, e}$ \\
\hline HOMA-IR & $2.0 \pm 2.1$ & $0.8 \pm 0.3$ & $8.1 \pm 8.6^{b}$ & $2.0 \pm 1.0^{d, e}$ \\
\hline Leptin $(\mathbf{n g} / \mathbf{m L})$ & $45.2 \pm 18.4$ & $3.2 \pm 2.4^{c}$ & $36.8 \pm 13.4$ & $16.4 \pm 10.5^{c, e}$ \\
\hline Adiponectin $(\mu \mathrm{g} / \mathrm{mL})$ & $2.9 \pm 1.1$ & $4.8 \pm 1.7^{c}$ & $8.1 \pm 5.5^{a}$ & $14.7 \pm 8.0 c, e$ \\
\hline C-Peptide (ng/mL) & $2.5 \pm 1.3$ & $1.4 \pm 1.0^{d}$ & $4.2 \pm 1.1^{a}$ & $2.0 \pm 0.3$ \\
\hline Triglyceride (mg/dL) & $116 \pm 82$ & $58 \pm 24^{c}$ & $175 \pm 59^{b}$ & $96 \pm 36^{c, f}$ \\
\hline Cholesterol (mg/dL) & $168 \pm 40$ & $192 \pm 48$ & $192 \pm 29^{b}$ & $155 \pm 23^{c, f}$ \\
\hline $\mathrm{HDL}(\mathrm{mg} / \mathrm{dL})$ & $44 \pm 18$ & $53 \pm 14^{d}$ & $44 \pm 11$ & $47 \pm 9$ \\
\hline $\mathrm{LDL}(\mathrm{mg} / \mathrm{dL})$ & $100 \pm 28$ & $127 \pm 41$ & $113 \pm 26$ & $89 \pm 20^{d, f}$ \\
\hline \multicolumn{5}{|l|}{ Thyroid Profile (n=9 pairs) } \\
\hline Triiodothyronine (T3; nmol/L) & $1.30 \pm 0.29$ & $0.74 \pm 0.16^{c}$ & $1.64 \pm 0.41$ & $1.53 \pm 0.23^{e}$ \\
\hline Thyroxin (T4; nmol/L) & $90.6 \pm 18.5$ & $82.3 \pm 17.5$ & $81.4 \pm 9.4$ & $82.2 \pm 9.0$ \\
\hline Thyroid stimulating hormone (TSH; mIU/L) & $1.3 \pm 1.0$ & $1.1 \pm 0.8$ & $0.7 \pm 0.5$ & $0.7 \pm 0.7$ \\
\hline Systolic BP (mm Hg) & $132 \pm 8$ & $118 \pm 11^{c}$ & $132 \pm 18$ & $122 \pm 13$ \\
\hline Diastolic BP (mm Hg) & $82 \pm 8$ & $72 \pm 10^{c}$ & $78 \pm 13$ & $75 \pm 12$ \\
\hline
\end{tabular}

Data are presented as mean $\pm \mathrm{SD}$.

$a_{p<0.01}$

${ }_{p}<0.05$ - denotes significant difference from BLC baseline

$c_{p}<0.01$,

$d_{p}<0.05$ - denotes significant difference from respective baseline

$e_{p}<0.01$

$f_{p}<0.05$ - denotes significant difference from BLC 7 month

HOMA-IR: homeostatic model assessment-estimated insulin resistance 